\title{
Ceftriaxone Treatment for Neuronal Deficits: A Histological and MEMRI Study in a Rat Model of Dementia with Lewy Bodies
}

\author{
Ying-Jui Ho $\mathbb{D}^{1},{ }^{1}$ Jun-Cheng Weng, ${ }^{2,3}$ Chih-Li Lin $\mathbb{D}^{4},{ }^{4}$ Mei-Shiuan Shen, ${ }^{1}$ Hsin-Hua Li, ${ }^{4}$ \\ Wen-Chieh Liao, ${ }^{5,6}$ Nu-Man Tsai, ${ }^{7}$ Ching-Sui Hung $\mathbb{D}^{8},{ }^{8}$ Te-Jen Lai $\mathbb{D}^{4},{ }^{4,9}$ and I-Yen Lee $\mathbb{i D}^{10}$ \\ ${ }^{1}$ Department of Psychology, Chung Shan Medical University Hospital, Chung Shan Medical University, Taichung 402, Taiwan \\ ${ }^{2}$ Department of Medical Imaging and Radiological Sciences, Chang Gung University, Taoyuan 33302, Taiwan \\ ${ }^{3}$ Department of Psychiatry, Chang Gung Memorial Hospital, Chiayi 613, Taiwan \\ ${ }^{4}$ Institute of Medicine, Chung Shan Medical University, Taichung 402, Taiwan \\ ${ }^{5}$ Department of Anatomy, Faculty of Medicine, Chung Shan Medical University, Chung Shan Medical University Hospital, \\ Taichung 402, Taiwan \\ ${ }^{6}$ Department of Pediatrics, Chung Shan Medical University Hospital, Taichung 402, Taiwan \\ ${ }^{7}$ School of Medical Laboratory and Biotechnology, Chung Shan Medical University, Taichung 402, Taiwan \\ ${ }^{8}$ Occupational Safety and Health Office, Taipei City Hospital, Taipei 10341, Taiwan \\ ${ }^{9}$ Department of Psychiatry, Chung Shan Medical University Hospital, Chung Shan Medical University, Taichung 402, Taiwan \\ ${ }^{10}$ Division of Urology, Department of Surgery, Tungs' Taichung Metroharbor Hospital, Taichung 43503, Taiwan
} Correspondence should be addressed to Ying-Jui Ho; joshuayjho@gmail.com, Ching-Sui Hung; bessyhung@gmail.com,
Te-Jen Lai; tejenlai@hotmail.com, and I-Yen Lee; homer9001065@gmail.com

Received 22 December 2017; Revised 22 January 2018; Accepted 12 March 2018; Published 1 August 2018

Academic Editor: Barbara Picconi

Copyright (C) 2018 Ying-Jui Ho et al. This is an open access article distributed under the Creative Commons Attribution License, which permits unrestricted use, distribution, and reproduction in any medium, provided the original work is properly cited.

Dementia with Lewy bodies (DLB) is characterized by neuronal deficits and $\alpha$-synuclein inclusions in the brain. Ceftriaxone (CEF), a $\beta$-lactam antibiotic, has been suggested as a therapeutic agent in several neurodegenerative disorders for its abilities to counteract glutamate-mediated toxicity and to block $\alpha$-synuclein polymerization. By using manganese-enhanced magnetic resonance imaging (MEMRI) and immunohistochemistry, we measured the effects of CEF on neuronal activity and $\alpha$-synuclein accumulation in the brain in a DLB rat model. The data showed that CEF corrected neuronal density and activity in the hippocampal CA1 area, suppressed hyperactivity in the subthalamic nucleus, and reduced $\alpha$-synuclein accumulation, indicating that CEF is a potential agent in the treatment of DLB.

\section{Introduction}

Dementia with Lewy bodies (DLB) is a common neurodegenerative dementia, accounting for approximately $10 \%-$ $25 \%$ of the dementia population. DLB patients show progressive cognitive decline and motor dysfunction. Psychiatric symptoms are reported in $99.2 \%$ of patients with DLB. DLB patients show higher subscores in the items of delusions, hallucinations, agitation, anxiety, irritation, and aberrant motor behavior, compared to the patients with Parkinson's disease dementia, which seriously affects their quality of life and social activities [1]. There is currently no rat model suitable for searching for more effective medications. In addition to the symptoms of progressive dementia and parkinsonism, accumulation of Lewy bodies in the central nervous system is the hallmark of DLB $[2,3]$. Lewy bodies are cytoplasmic eosinophilic protein inclusions of $\alpha$-synuclein ( $\alpha$-syn) [4-6]. $\beta$-Amyloid (A $\beta$ ) suppresses the clearance of $\alpha$-syn $[7,8]$ and accelerates oligomerization of $\alpha$-syn and its toxicity, leading to the deterioration caused by $\alpha$-syn [9]. Moreover, direct or indirect interactions of $\alpha$-syn and A $\beta$ promote their mutual aggregation and accumulation, disturb the function 
of mitochondria, cause excessive glutamate release in the synapse, and eventually result in excitotoxicity and cell death in the brain [8]. That is, $\mathrm{A} \beta$ aggravates the neurotoxicity of $\alpha$-syn in the brain [10].

Viral vectors carrying the SNCA gene can constantly express $\alpha$-syn in the host. Some studies have injected these vectors into the hippocampus, cortex, and striatum of rats to model the pathological changes seen in DLB $[9,11]$. The advantages of using viral vectors are low cost, fast model establishment, and high expression of genes. Additionally, specific regions of the brain that are of research interest may be targeted [12]. Although viral vectors can deliver specific genes to the brain tissues of the host, the use of the method to transfer genes to larger brain regions, such as the cortex, remains a challenge $[13,14]$. In the present study, the $\alpha$-syn gene vector was injected into the lateral ventricle of rats, which diffused with the flow of the cerebrospinal fluid, facilitated $\alpha$-syn expression throughout the regions of the brain, and contributed to the establishment of a rat model for DLB.

There is currently no specific medicine for treating DLB. Because of the cognitive dysfunction in patients with DLB, memantine, an antagonist of N-methyl-D-aspartate (NMDA) receptors, is used to alleviate this symptom. This indicates the involvement of glutamatergic hyperactivity in the disease. Rothstein and colleagues found that ceftriaxone (CEF) increases the expression of glutamate transporter-1 (GLT-1), which can remove excessive glutamate released in the synaptic cleft, reduce excitotoxicity, and yield neuroprotective effects [15]. We have demonstrated that CEF treatment prevents the decrease of neuronal activity [16] and loss of neuronal density in the nigrostriatal system and in the hippocampus in a Parkinson's disease (PD) rat model $[17,18]$. Moreover, an in vitro model of PD revealed that CEF binds with high affinity to $\alpha$-syn and blocks its polymerization [19].

Manganese-enhanced magnetic resonance imaging (MEMRI) is a valuable imaging tool for directly measuring activity-dependent neuronal events in a living animal [20]. Manganese ion $\left(\mathrm{Mn}^{2+}\right)$ may enter neurons through voltage-gated calcium $\left(\mathrm{Ca}^{2+}\right)$ channels [21] where it is retained there with a biological half-life of 51-74 days according to a study of adult rats' brains [22]. Before MR imaging, the animal was injected intraperitoneally (i.p.) with $\mathrm{MnCl}_{2}$, and its neurons took up $\mathrm{Mn}^{2+}$ when activated. A quantitative analysis indicated that $\mathrm{Mn}^{2+}$ accumulates in neurons according to their activity, because $\mathrm{Ca}^{2+}$ elevation is related to the firing activity in excitatory neurons [23]. In addition, its paramagnetic feature enables $\mathrm{Mn}^{2+}$ to shorten the longitudinal relaxation time (T1) of water protons and thus enhance the T1-weighted MR signal that is specific to the tissues where $\mathrm{Mn}^{2+}$ has accumulated [24], thus rendering it an excellent MRI-detectable contrast agent [25]. Therefore, tissue contrast in $\mathrm{Mn}^{2+}$-induced $\mathrm{T} 1$ signal intensity in MEMRI may be contingent upon the differential accumulation of $\mathrm{Mn}^{2+}$ in active and silent brain regions in which topography can be absolutely quantified by measuring the absolute $\mathrm{T} 1$ (or $\mathrm{R} 1=\mathrm{T} 1^{-1}$ ) value $[23,26]$.

When combined with pharmacological manipulation, MEMRI can be used to detect changes in neuronal activities that are produced by the treatment. However, whether changes of neuronal activity are accompanied by a loss of neurons in the DLB rat model remains unclear. The purpose of this study was to elucidate the effects of CEF $(100 \mathrm{mg} / \mathrm{kg} /$ day) on neuronal density and activity in the brain of the DLB rat model using immunohistochemistry and MEMRI.

\section{Materials and Methods}

2.1. Animals. Twelve-week-old male Wistar rats $(420 \pm 30 \mathrm{~g}$; $n=25$; BioLASCO Taiwan Co Ltd., ROC) were randomly assigned to groups of 3 or 4 and housed in acrylic cages $\left(35 \times 56 \times 19 \mathrm{~cm}^{3}\right)$ in a temperature-controlled animal room $\left(21-25^{\circ} \mathrm{C}\right)$ with free access to food and water. Photoperiods in rodent rooms were controlled using an automatic timer set to provide $12 \mathrm{~h}$ of light (from 7:00 to 19:00) and $12 \mathrm{~h}$ of dark (from 19:00 to 7:00). To minimize defensive behaviors and stress response to the experimenter, prior to the start of the experiment, all animals were handled for $5 \mathrm{~min} /$ day on 2 consecutive days. All experimental procedures were performed according to the NIH Guide for the Care and Use of Laboratory Animals and were approved by the Animal Care Committee of Chung Shan Medical University (IACUC approval number 1455). All efforts were made to minimize animal suffering and to reduce the number of animals used [27].

2.2. General Procedures. For inducing DLB in the rat model, rats were randomly divided into three groups and underwent stereotaxic brain surgery on day 0 as described in our previous reports $[8,27]$. Briefly, the rats were anesthetized by an i.p. injection of Zoletil $(20 \mathrm{mg} / \mathrm{kg}$; Virbac, Carros, France) and mounted in a stereotaxic frame. To overexpress $\alpha$-syn, recombinant adeno-associated viral (rAAV) vector containing human $\alpha$-syn gene, SNCA, $(10 \mu \mathrm{g} / 10 \mu \mathrm{L} / \mathrm{rat})$ was injected into the left lateral cerebral ventricle using the following coordinates adapted from the rat brain atlas: AP: $-0.8 \mathrm{~mm}$, ML: $-1.5 \mathrm{~mm}$, and DV: $-3.6 \mathrm{~mm}$ from the bregma, midline, and skull surface, respectively. The $\mathrm{A} \beta_{1-42}$ solution $(5 \mu \mathrm{g} / 2.5 \mu \mathrm{L} /$ side $\times 2$ sides/rat $)$ was bilaterally infused in the prefrontal cortex using the following stereotaxic coordinates: AP: $1.6 \mathrm{~mm}, \mathrm{ML}: \pm 2.0 \mathrm{~mm}$, and DV: $-2 \mathrm{~mm}$ from the bregma, midline, and skull surface, respectively. During the 5 days after surgery, the rats were housed individually in plastic cages and $10 \%$ sucrose solution was provided ad libitum to prevent weight loss after surgery and reduce mortality.

Starting from the next day after the surgery (day 1 ), the animals received the following treatments: the "sham + saline" group $(n=5)$ and "DLB + saline" group $(n=7)$ were injected with saline $(1 \mathrm{~mL} / \mathrm{kg} /$ day, i.p.), and the "DLB + CEF" group $(n=6)$ was injected with ceftriaxone (CEF) ( $100 \mathrm{mg} / \mathrm{kg} /$ day, i.p.) (Roche, Kaiseraugst, Switzerland) for 27 days.

Hydrated manganese chloride $\left(\mathrm{MnCl}_{2} \cdot 4 \mathrm{H}_{2} \mathrm{O}\right.$, Sigma Aldrich, UK) was dissolved in saline at a concentration of $100 \mu \mathrm{mol} / \mathrm{mL}(20 \mathrm{mg} / \mathrm{mL})$. On day 26 , all rats received two $1 \mathrm{~mL} / \mathrm{kg}$ i.p. injections of $\mathrm{MnCl}_{2}$ solution separated by $1 \mathrm{~h}$ (total dose $40 \mathrm{mg} / \mathrm{kg}$ ) [28]. Twenty-four hours later 
(day 27), when $\mathrm{Mn}^{2+}$-induced signal enhancement reached a stable asymptotic level [28], the rats were transported to the MR center for MR imaging, anesthetized during imaging, and transported back to the animal room.

On day 28 , rats were euthanized by exposure to $\mathrm{CO}_{2}$, transcardially perfused with phosphate-buffered saline (PBS) followed by $4 \%$ paraformaldehyde in PBS. The brain was then immediately removed and postfixed in PBS containing $30 \%$ sucrose and $4 \%$ paraformaldehyde at $4{ }^{\circ} \mathrm{C}$ until use.

2.3. MEMRI Data Acquisition. Brain images were acquired on a 7T MRI system (Bruker BioSpec, Karlsruhe, Germany), as described in our previous paper [16]. Briefly, rats were initially anesthetized with $5 \%$ isoflurane vapor in oxygen at a flow rate of $500 \mathrm{~mL} / \mathrm{min}$. T2-weighted contrast of anatomical images was acquired using the turbo rapid acquisition with relaxation enhancement sequence. T1-weighted images were acquired using a multislice spin-echo sequence. To improve detection sensitivity over the full extent of $\mathrm{Mn}^{2+}$ concentrations, R1 images were acquired using a rapid acquisition with relaxation enhancement with variable time of repetition. Six sets of images corresponding to six TRs (ranging from 500 to $3500 \mathrm{~ms}$ ) were taken during the recovery of the longitudinal magnetization to perform R1 mapping. The R1 maps shown in the paper are representations of the distribution of $\mathrm{Mn}^{2+}$ uptake in the region of interest (ROI) that were manually defined in the hippocampus and subthalamic nucleus (STN) in the coronal images. The mean signal intensity for all voxels in the ROIs, averaged for both hemispheres, was used to compare R1 differences between the groups.

2.4. Histological Assessment. For histological assessment, frozen coronal sections of the brain ( $25 \mu \mathrm{m}$ thick) were cut and mounted on gelatinized slides and maintained in PBS until staining. The regions seen in the stained brain sections were identified according to the rat brain atlas [29] and used for image analysis to measure histological changes, as described previously $[17,28,30-34]$. The ROI was defined in the hippocampus (bregma $-2.76 \mathrm{~mm}$ to $-4.20 \mathrm{~mm}$ ).

Nissl staining, which is used to identify pyramidal cells in the hippocampus, was performed as described in our previous reports $[16,17,32]$. $\alpha$-Syn staining was used to evaluate the density of $\alpha$-syn immune-positive cells in the brain sections $[8,35]$. The coronal sections were rinsed $(3 \times 5 \mathrm{~min}$ each) with $0.05 \mathrm{M}$ Tris buffer (TB), incubated for $20 \mathrm{~min}$ at room temperature with $0.3 \%$ hydrogen peroxide to block endogenous peroxidase activity, and blocked by incubation for $1 \mathrm{~h}$ at room temperature in 100\% normal goat serum dissolved in TB containing 10\% Triton-100. They were then incubated overnight at $4^{\circ} \mathrm{C}$ with polyclonal rabbit anti- $\alpha$ syn antibody $(1: 100$; GeneTex, USA) and incubated sequentially for $2 \mathrm{~h}$ at room temperature with HRP-conjugated goat anti-rabbit IgG ( $1: 200$; GeneTex, USA), followed by $5 \mathrm{~min}$ at room temperature with $3,3^{\prime}$-diaminobenzidine tetrachloride (DAB; Sigma, USA) before being dehydrated in ethanol and xylene. The stained sections of images were acquired using a microscope (ZEISS AXioskop 2, Germany). The density of neurons and $\alpha$-syn-positive cells (number $/ \mathrm{mm}^{2}$ ) in the brain sections were counted using the Image Pro Plus Software 6.0 (Media Cybernetics, CA, USA) as described in our previous paper [16].

2.5. Data Analysis. SPSS 17.0 statistical software was used for data analysis. Analysis of variance (ANOVA) and the leastsignificant difference (LSD) post hoc test were used to analyze the data. All results are expressed as the mean \pm standard error of the mean (SEM). The level of significance was defined as $P<0.05$.

\section{Results}

3.1. Image Analysis. One-way ANOVA revealed significant differences in brain MRI images between the groups. Compared with the sham + saline group, the DLB + saline group showed decreased $\mathrm{R} 1, \mathrm{Mn}^{2+}$ uptake level in the hippocampal CA1 area $(F(2,17)=3.79, P=0.05)$ (LSD post hoc test, $P$ value $=0.03)$. However, normal $\mathrm{Mn}^{2+}$ uptake in the hippocampal CA1 area was seen after CEF treatment because no difference between the sham + saline and DLB + CEF groups was found (Figure 1). One-way ANOVA revealed significant differences in STN MRI images between the groups $(\mathrm{F}(2,17)=5.57, P=0.016)$. The LSD post hoc test revealed a higher $\mathrm{Mn}^{2+}$ uptake and neuronal activity in the DLB + saline group, compared with the sham + saline group $(P<0.05)$. The rats receiving CEF treatment showed the same neural activity in the STN as did the controls (Figure 2).

\subsection{Histological Assay}

3.2.1. Density of Pyramidal Neurons in the Hippocampal CA1. The ANOVA revealed that density of pyramidal neurons in the hippocampal CA1 showed a significant difference $(F(2,14)=56.69, P<0.001)$, and post hoc analysis showed that rats in the DLB + saline group had a significantly lower neuronal density than did those in the sham + saline group $(P<0.001)$. The DLB + CEF group showed a higher neuronal density than the DLB + saline group $(P<0.001)$ (Figure 3$)$.

3.2.2. Density of $\alpha$-Syn in the Dentate Gyrus. The ANOVA revealed significant differences in the density of $\alpha$-synpositive cells in the hippocampal dentate gyrus (DG) $(F(2,14)=3.91, P<0.05) \quad($ partial eta squared $=0.638)$, and post hoc analysis showed that rats in the DLB + saline group $\left(152 \pm 56\right.$ number $\left./ \mathrm{mm}^{2}\right)$ had a significantly higher density of $\alpha$-syn-positive cells compared with the sham + saline group $\left(23 \pm 10\right.$ number $\left./ \mathrm{mm}^{2}\right) \quad(P<0.05)$. The DLB + CEF group $\left(90 \pm 32\right.$ number $\left./ \mathrm{mm}^{2}\right)$ showed no difference in the density of $\alpha$-syn-positive cells compared with the sham + saline group (Figure 4).

\section{Discussion}

In the DLB rat model, we found an accumulation of $\alpha$-syn in the hippocampal DG and lowered neuronal density and activity in the hippocampal CA1 of the DLB + saline group compared with the sham + saline group. In addition, hyperactivity in the STN was also observed in DLB + saline rats. Treatment with CEF at a dose of $100 \mathrm{mg} / \mathrm{kg}$ corrected these 


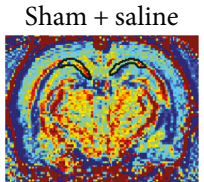

(a)

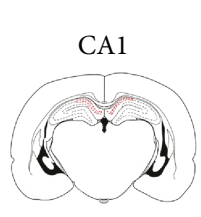

(d)

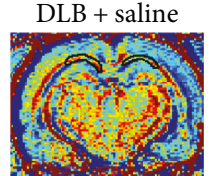

(b)

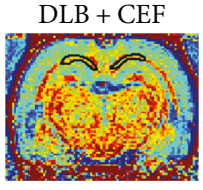

(c)

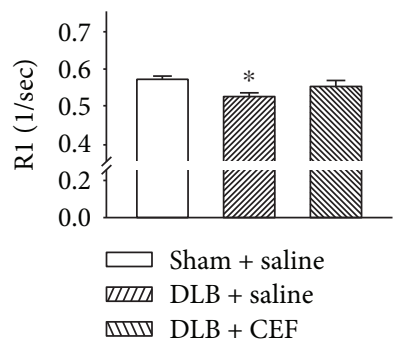

(e)

FIGURE 1: Effect of treatment with ceftriaxone (CEF) on neuronal activity in the hippocampal CA1 area of the DLB rat model. The rats were bilaterally infused with $A \beta_{1-42}$ in the prefrontal cortex and unilaterally infused with viral vectors with the SNCA gene in the left lateral ventricle to model DLB in rats. The sham + saline group and DLB + saline group were injected with saline ( $1 \mathrm{~mL} / \mathrm{kg} / \mathrm{day}$, i.p.), and the $\mathrm{DLB}+\mathrm{CEF}$ group was injected with CEF $(100 \mathrm{mg} / \mathrm{kg} /$ day, i.p.) for 27 days. (a-c) Coronal R1 maps of the rat brain. The regions of interest used for quantitative analysis of $\mathrm{Mn}^{2+}$-induced signal enhancement in the hippocampal CA1 area are indicated by the outlines on the schematic (d). (e) Quantitative results. Data are expressed as the mean \pm SEM. ${ }^{*} P<0.05$, compared with the sham + saline group.

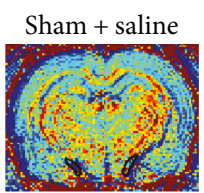

(a)

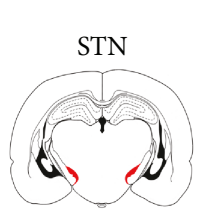

(d)

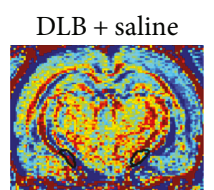

(b)

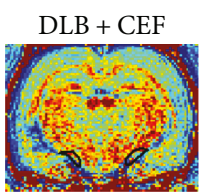

(c)

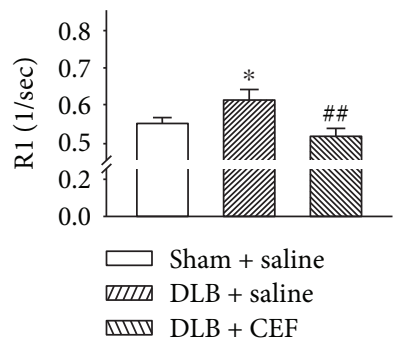

(e)

Figure 2: Effect of treatment with ceftriaxone (CEF) on neuronal activity in the subthalamic nucleus (STN) in the DLB rat model. The group names are the same as those in Figure 1. $(\mathrm{a}-\mathrm{c})$ Coronal R1 maps of the rat brain. The regions of interest used for quantitative analysis of $\mathrm{Mn}^{2+}$-induced signal enhancement in the STN are indicated by the outlines on the schematic (d). (e) Quantitative results. Data are expressed as the mean \pm SEM. ${ }^{*} P<0.05$, compared with the sham + saline group; ${ }^{\# \# ~} P<0.01$, compared with the DLB + saline group.

neuronal deficits. CEF was previously found to increase GLT1 expression, resulting in sequestration of excess synaptic glutamate and protection of hippocampal neurons from excitotoxicity [36]. It has been reported that treatment with CEF prevented neurodegeneration in the hippocampus and nigrostriatal system, increasing neuronal activity [16] and improving cognitive function in an MPTP-induced PD rat model $[17,18]$. We have previously reported that CEF, at the dosages of 100 and $200 \mathrm{mg} / \mathrm{kg}$, per se did not affect motor function, cognitive behavior, and neuronal density in the hippocampus in healthy rats [17]. These results suggest that CEF can prevent DLB-related neuronal deficits in the brain.
The present study revealed that the injection of $\mathrm{A} \beta$ and viral vectors with the SNCA gene into the brain caused an accumulation of $\alpha$-syn, neuronal loss, and lowered neuronal activity in the hippocampus, suggesting that this method can induce a useful model of DLB. The pathophysiology of DLB is related to the aggregation of Lewy bodies and Lewy neurites that are formed by $\alpha$-syn accumulation [37, 38], resulting in neurotoxicity and cell loss in the limbic system, brainstem, and cortical regions $[5,39,40]$. Although $\alpha$-syn accumulation is the core pathological feature of DLB, the deposition of extracellular $A \beta$ is also observed in the brains of up to $80 \%$ of DLB patients [7]. Direct and indirect 


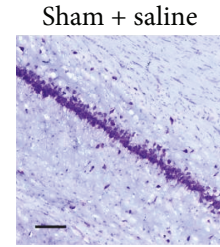

(a)

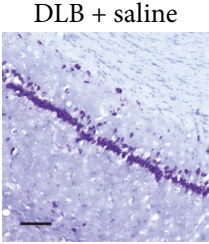

(b)

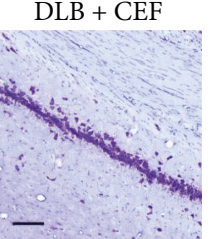

(c)
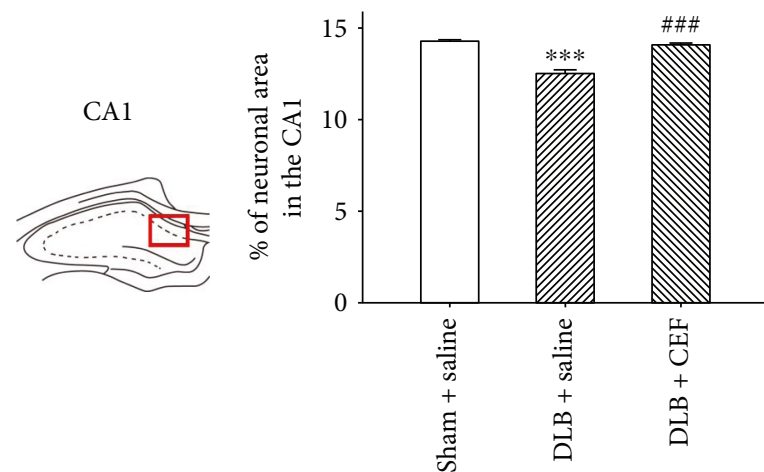

(d)

(e)

FIgURE 3: Effect of ceftriaxone (CEF) on the neuronal density in the hippocampal CA1 area of DLB rats. The treatment and group names are the same as those in Figure 1. $(\mathrm{a}-\mathrm{c})$ Coronal brain sections of the hippocampal CA1 area; pyramidal cells are revealed through Nissl staining. Magnification, 200x; bar, $100 \mu \mathrm{m}$. (d) CA1 region of the hippocampus. (e) Quantitative results. Data are expressed as mean \pm SEM. ${ }^{* * *} P<0.001$, compared with the sham + saline group; ${ }^{\# \# \# ~} P<0.001$, compared with the DLB + saline group.

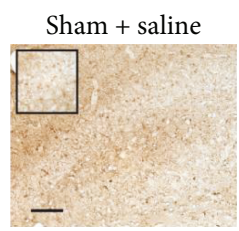

(a)

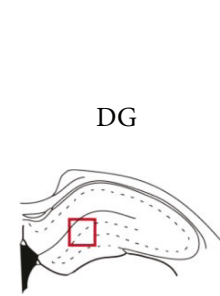

(d)

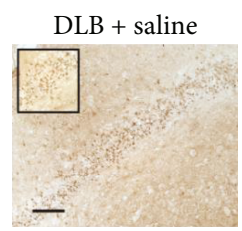

(b)

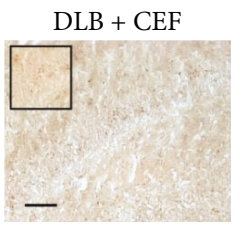

(c)

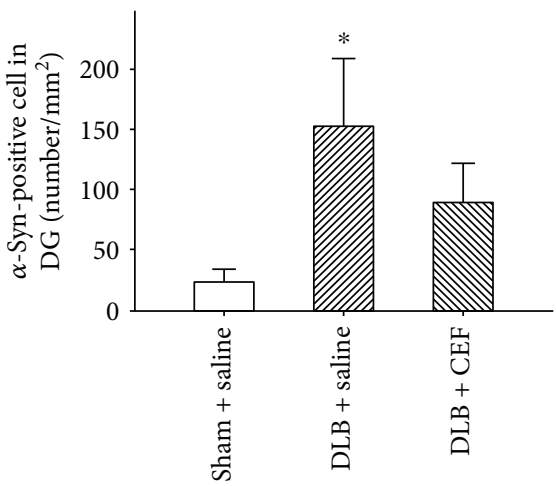

(e)

Figure 4: Effect of ceftriaxone (CEF) on the density of $\alpha$-synuclein- ( $\alpha$-syn-) positive cells in the DG of DLB rats. The treatment and group names are the same as those in Figure 1. $(\mathrm{a}-\mathrm{c})$ Coronal sections in the DG. $\alpha$-Syn-positive cells are indicated by anti- $\alpha$-syn labeling in representative coronal sections. Magnification, 200x; bar, $100 \mu \mathrm{m}$. High magnification images (1000x) of $\alpha$-syn-positive cells are shown in the insets. (d) The DG of the hippocampus. (e) Quantitative results. Data are expressed as the mean \pm SEM. ${ }^{*} P<0.05$, compared with the sham + saline group.

interaction between $\alpha$-syn and $\mathrm{A} \beta$ has been reported to promote $\alpha$-syn aggregation [8]. A $\beta$ increases the toxicity of $\alpha$-syn [8] and aggravates the relevant defects triggered by $\alpha$-syn, causing neurodegeneration [41]. Thus, in addition to injecting the $\alpha$-syn gene vector into the rat's lateral ventricle, $A \beta$ was also injected into the bilateral prefrontal cortex to more accurately model the pathological characteristics of DLB. In this study, the $\alpha$-syn gene vector was injected into the lateral ventricle of rats, which diffused with the flow of the cerebrospinal fluid and facilitated the expression of 
$\alpha$-syn throughout the regions of the brain. A high level of $\alpha$ syn accumulation was observed in the hippocampal DG area of DLB + saline rats, which may be involved in lower density of pyramidal neurons in the hippocampal CA1 because the abnormal accumulation of $\alpha$-syn in the limbic system leads to cell loss and dysfunction in the hippocampus [42]. Reportedly, CEF binds to $\alpha$-syn [19] and may thus block polymerization of $\alpha$-syn and exert neuroprotective effects in vitro [19], which may underlie the effects of CEF on reducing $\alpha$-syn accumulation and restoring neuronal density and activity in the hippocampus of DLB rats. The subject number in the study is relatively small. The level of $\alpha$-syn in the DG of the hippocampus in the DLB + CEF group may be only partially reversed. To increase statistical power and to provide convincing treatment effect, further study using a larger number of subject is needed.

$\mathrm{A} \beta$ oligomers have been reported to play a pivotal role in synaptic impairment and neuronal degeneration [43]. Based on this amyloid hypothesis, the formation of soluble species of $\mathrm{A} \beta$ can directly interfere with memory formation and cause synaptic degradation, excitotoxicity, and cell death [44], leading to cognitive decline. Glutamate is one of the main excitatory neurotransmitters in the central nervous system, is involved in synaptic plasticity, memory, and learning, and plays a substantial role in the pathogenesis from the early stages of neurodegenerative diseases. To bind $\mathrm{A} \beta$ peptides, NMDA receptors have attracted considerable interest because of their toxic effects and involvement in neurodegeneration [45]. $\mathrm{A} \beta$ oligomers interfere with glutamatergic transmission. Under pathological conditions, elevation of $\mathrm{A} \beta$ levels blocks glutamate uptake at the synaptic cleft, leading to increased glutamate levels, stimulation of NMDA receptors [46], and activation of extrasynaptic NR2B-enriched NMDA receptors [45], causing an increase of intracellular calcium levels and the activation of metabolic pathways responsible for neuronal shrinkage, synaptic loss, and neurodegeneration [47]. Moreover, A $\beta$ oligomers form complexes with alpha7-nicotinic receptors at presynaptic sites, causing increased levels of glutamate release, and they are involved in synaptic plasticity [48]. These factors indicate that $\mathrm{A} \beta$ is responsible for impairments of synaptic plasticity, cell loss, and neuronal death.

Glutamatergic hyperactivity can cause neurodegeneration $[49,50]$ and may thus be involved in the parkinsonism of DLB. STN neurons express glutamate, innervate the substantia nigra (SNc), and are hyperactivated in PD. Modulation of STN activity by deep brain stimulation improves parkinsonism, indicating that STN hyperactivity is associated with the development of parkinsonian features [51, 52]. STN hyperactivation increases glutamate release, causing excitotoxicity of dopaminergic neurons in the SNc [53]. In the present MEMRI-based study, we observed hyperactivity in the STN in the DLB + saline rats and found that this change was prevented with CEF treatment. This was consistent with our previous findings that CEF prevents dopaminergic and hippocampal degeneration in a PD rat model $[17,18]$. We therefore suggest that CEF treatment reduced STN hyperactivity by increasing GLT-1 expression and glutamate reuptake and thus blocked neurodegeneration in the DLB rat model.
The risk of developing dementia in patients with DLB may increase with hippocampal cell loss, because the decline in cognitive function is associated with hippocampal degeneration [54]. Since that hippocampal CA1 is reported to be involved in working memory and cognition [55] and that neurogenesis is observed in the DG [56], dysfunction of the hippocampus may result in impairments of memory and recognition $[57,58]$. The hippocampus shows vulnerability of excitotoxic damage due to rich of glutamatergic synapses [36]; excessive glutamate release and excitotoxicity-induced neurodegeneration in this region may lead to memory and recognition impairment, as seen in patients with DLB. The present study revealed hippocampal lesions in the CA1 of DLB rats, which was accompanied by lowered neuronal activity, as measured using MEMRI. Our another study found impairments in cognitive behaviors, for example, object recognition and avoidance learning, in the DLB rats, where deficits were improved after CEF treatment. (Data have been submitted for publication.) Regarding the clinical features of patients with DLB, in addition to cognitive dysfunction, motor impairment is also observed. However, in the experiment, the DLB rats did not exhibit motor dysfunction (data not shown). According to the "1-year rule," the consensus guidelines to distinguish DLB and PD dementia [59], if cognitive impairment occurs within 1 year following the onset of motor impairment or if cognitive impairment occurs earlier than motor impairment, DLB is diagnosed. Lack of motor impairment in the present study may be due to the short experimental period (only 28 days). The present histological findings showing increased $\alpha$-syn-positive cells in the hippocampus of DLB rats are in line with that $\alpha$-syn pathologies observed in the hippocampus of patients with DLB, which is associated with memory impairment [60]. Furthermore, increasing evidence supports that $\alpha$-syn aggregates may be the real culprit, causing deficits in neurotransmission and neurogenesis in the hippocampus, which is considered to be involved in mechanisms for the hippocampal dysfunctions and associated neuropsychiatric manifestations in synucleinopathy [61]. CEF treatment prevented decreased neuronal density and activity in the area, indicating that CEF may have the potential for treating neurodegeneration and dementia in patients with DLB [17].

Increase of GLT-1 expression may be involved in the CEF-induced prevention of the loss of neuronal function in DLB rats. Excessive release of glutamate and hyperactivity of the glutamatergic system play a critical role in neuronal and behavioral symptoms in neurodegenerative diseases [62, 63]. GLT-1 is responsible for glutamate clearance [64-67] and thus prevents glutamate excitotoxicity [68-70]. In previous studies $[17,18]$, we found that CEF treatment caused a dose-dependent increase in GLT-1 expression in the brain, had a neuroprotective effect in the hippocampus and nigrostriatal dopaminergic system, and improved cognitive function. We thus suggest that upregulation of GLT-1 and reduction of excitotoxicity may underlie the neuronal protective effect of CEF. Further studies are needed to elucidate the downstream molecular pathway of $\mathrm{CEF}$ that modulates glutamate transmission and their neuroprotective effects. 
MEMRI data associated with $\mathrm{Mn}^{2+}$ uptake are used to quantitatively evaluate neural activity, where R1 value represents the accumulation of $\mathrm{Mn}^{2+}$ in neurons [23]. Consistently, our data showed that MEMRI R1 values in the hippocampus reflect cell loss in the DLB rats and treatment effects of CEF against the disease [16]. Higher neuronal activities in the STN of the DLB + saline group indicated similar clinical and pathological characteristics associated with parkinsonism and neurodegeneration [71]. Elevation of STN activities (i.e., a burst pattern of neuronal firing) is also observed in patients with PD [72]. Our previous study found a significant positive correlation between MEMRI R1 and neuronal density in the hippocampus [16], indicating that $\mathrm{R} 1$ values in the hippocampus may serve as an indicator of neuronal density of the hippocampus in the DLB rat model. Although the present data revealed hippocampal lesions with abnormal neuronal activity 4 weeks after the surgery, it would be of interest to measure the changes long after the surgery, which would provide evidence to elucidate whether the DLB rat model shares the same progressive features as in patients with DLB.

In conclusion, the results demonstrated that CEF treatment prevented the loss of neuronal density and activity in the hippocampus and also normalized the hyperactivity of the STN in the DLB rat model. Treatment with CEF prevented the accumulation of $\alpha$-syn in the hippocampus. Moreover, the MEMRI R1 value may serve as a suitable indicator for DLB severity and treatment effect. Our data provide support for the use of CEF as a potential therapeutic agent to prevent dementia in patients with DLB.

\section{Disclosure}

Some of the data in this paper have been presented as conference posters.

\section{Conflicts of Interest}

The authors declare no conflicts of interest for the material in the manuscript.

\section{Acknowledgments}

This work was supported by grants from the Ministry of Science and Technology (106-2410-H-040-003-MY2, 104-2923H-040-001-MY3, 104-2314-B-040-007-MY2, 104-2628-E040-001-MY2, 106-2320-B-040-021-MY3, and 104-2628E-182-004-MY2), Chung Shan Medical University Hospital (CSH-2017-C-007), Taipei City Government, Taiwan (10501-62-046). The authors appreciate Dr. Pai-Yi Chiu's contribution of critical review of this paper. Microscopy and cryosectioning were performed in the Instrument Center of Chung Shan Medical University, which is supported by the MOST, Ministry of Education, and Chung Shan Medical University. The authors appreciate the full support from the Instrumentation Center and Department of Electrical Engineering, National Taiwan University, Taiwan.

\section{References}

[1] P. Y. Chiu, C. T. Tsai, P. K. Chen, W. J. Chen, and T. J. Lai, "Neuropsychiatric symptoms in Parkinson's disease dementia are more similar to Alzheimer's disease than dementia with Lewy bodies: a case-control study," PLoS One, vol. 11, no. 4, article e0153989, 2016.

[2] H. Oda, Y. Yamamoto, and K. Maeda, "Neuropsychological profile of dementia with Lewy bodies," Psychogeriatrics, vol. 9, no. 2, pp. 85-90, 2009.

[3] J. Macijauskienè and V. Lesauskaite, "Dementia with Lewy bodies: the principles of diagnostics, treatment, and management," Medicina, vol. 48, no. 1, pp. 1-8, 2012.

[4] M. G. Spillantini, R. A. Crowther, R. Jakes, M. Hasegawa, and M. Goedert, " $\alpha$-Synuclein in filamentous inclusions of Lewy bodies from Parkinson's disease and dementia with Lewy bodies," Proceedings of the National Academy of Sciences of the United States of America, vol. 95, no. 11, pp. 6469-6473, 1998.

[5] W. S. Kim, K. Kågedal, and G. M. Halliday, "Alpha-synuclein biology in Lewy body diseases," Alzheimer's Research \& Ther$a p y$, vol. 6, no. 5-8, p. 73, 2014.

[6] K. Beyer, M. Domingo-Sabat, and A. Ariza, "Molecular pathology of Lewy body diseases," International Journal of Molecular Sciences, vol. 10, no. 3, pp. 724-745, 2009.

[7] S. E. Marsh and M. Blurton-Jones, "Examining the mechanisms that link $\beta$-amyloid and $\alpha$-synuclein pathologies," Alzheimer's Research \& Therapy, vol. 4, no. 2, p. 11, 2012.

[8] C.-L. Lin, Y.-S. Cheng, H.-H. Li et al., “Amyloid- $\beta$ suppresses AMP-activated protein kinase (AMPK) signaling and contributes to $\alpha$-synuclein-induced cytotoxicity," Experimental Neurology, vol. 275, Part 1, pp. 84-98, 2016.

[9] C. R. Overk, A. Cartier, G. Shaked et al., "Hippocampal neuronal cells that accumulate $\alpha$-synuclein fragments are more vulnerable to $\mathrm{A} \beta$ oligomer toxicity via mGluR5 - implications for dementia with Lewy bodies," Molecular Neurodegeneration, vol. 9, no. 1, p. 18, 2014.

[10] C.-C. Chang, H.-H. Li, Y.-T. Chang et al., "A $\beta$ exacerbates $\alpha$ synuclein-induced neurotoxicity through impaired insulin signaling in $\alpha$-synuclein-overexpressed human SK-N-MC neuronal cells," CNS Neuroscience \& Therapeutics, vol. 24, no. 1, pp. 47-57, 2018.

[11] Y. Lim, V. M. Kehm, E. B. Lee et al., " $\alpha$-Syn suppression reverses synaptic and memory defects in a mouse model of dementia with Lewy bodies," Journal of Neuroscience, vol. 31, no. 27, pp. 10076-10087, 2011.

[12] H. Mochizuki, M. Yamada, and Y. Mizuno, " $\alpha$-Synuclein overexpression model," Journal of Neural Transmission Supplementa, vol. 70, pp. 281-284, 2006.

[13] P. Aldrin-Kirk, M. Davidsson, S. Holmqvist, J. Y. Li, and T. Björklund, "Novel AAV-based rat model of forebrain synucleinopathy shows extensive pathologies and progressive loss of cholinergic interneurons," PLoS One, vol. 9, no. 7, article e100869, 2014.

[14] M. F. Chesselet, "In vivo alpha-synuclein overexpression in rodents a useful model of Parkinson's disease," Experimental Neurology, vol. 209, no. 1, pp. 22-27, 2008.

[15] J. D. Rothstein, S. Patel, M. R. Regan et al., “ $\beta$-Lactam antibiotics offer neuroprotection by increasing glutamate transporter expression," Nature, vol. 433, no. 7021, pp. 73-77, 2005. 
[16] J. C. Weng, M. A. Tikhonova, J. H. Chen et al., "Ceftriaxone prevents the neurodegeneration and decreased neurogenesis seen in a Parkinson' disease rat model: an immunohistochemical and MRI study," Behavioural Brain Research, vol. 305, pp. 126-139, 2016.

[17] C. Y. Hsu, C. S. Hung, H. M. Chang, W. C. Liao, S. C. Ho, and Y. J. Ho, "Ceftriaxone prevents and reverses behavioral and neuronal deficits in an MPTP-induced animal model of Parkinson's disease dementia," Neuropharmacology, vol. 91, pp. 43-56, 2015.

[18] S. C. Ho, C. C. Hsu, C. R. Pawlak et al., "Effects of ceftriaxone on the behavioral and neuronal changes in an MPTP-induced Parkinson's disease rat model," Behavioural Brain Research, vol. 268, pp. 177-184, 2014.

[19] P. Ruzza, G. Siligardi, R. Hussain et al., "Ceftriaxone blocks the polymerization of $\alpha$-synuclein and exerts neuroprotective effects in vitro," ACS Chemical Neuroscience, vol. 5, no. 1, pp. 30-38, 2013.

[20] Y. J. Lin and A. P. Koretsky, "Manganese ion enhances T1weighted MRI during brain activation: an approach to direct imaging of brain function," Magnetic Resonance in Medicine, vol. 38, no. 3, pp. 378-388, 1997.

[21] A. Takeda, "Manganese action in brain function," Brain Research Reviews, vol. 41, no. 1, pp. 79-87, 2003.

[22] A. Takeda, J. Sawashita, and S. Okada, "Biological half-lives of zinc and manganese in rat brain," Brain Research, vol. 695, no. 1, pp. 53-58, 1995.

[23] S. Kikuta, Y. Nakamura, Y. Yamamura et al., "Quantitative activation-induced manganese-enhanced MRI reveals severity of Parkinson's disease in mice," Scientific Reports, vol. 5, no. 1, article 12800, 2015.

[24] O. Eschenko, S. Canals, I. Simanova, M. Beyerlein, Y. Murayama, and N. K. Logothetis, "Mapping of functional brain activity in freely behaving rats during voluntary running using manganese-enhanced MRI: implication for longitudinal studies," NeuroImage, vol. 49, no. 3, pp. 2544-2555, 2010.

[25] A. C. Silva and N. A. Bock, "Manganese-enhanced MRI: an exceptional tool in translational neuroimaging," Schizophrenia Bulletin, vol. 34, no. 4, pp. 595-604, 2008.

[26] J. C. Weng, J. H. Chen, P. F. Yang, and W. Y. I. Tseng, "Functional mapping of rat barrel activation following whisker stimulation using activity-induced manganese-dependent contrast," NeuroImage, vol. 36, no. 4, pp. 1179-1188, 2007.

[27] M. J. Castelhano-Carlos and V. Baumans, "The impact of light, noise, cage cleaning and in-house transport on welfare and stress of laboratory rats," Laboratory Animals, vol. 43, no. 4, pp. 311-327, 2009.

[28] S. J. Jackson, R. Hussey, M. A. Jansen et al., "Manganeseenhanced magnetic resonance imaging (MEMRI) of rat brain after systemic administration of $\mathrm{MnCl}_{2}$ : hippocampal signal enhancement without disruption of hippocampus-dependent behavior," Behavioural Brain Research, vol. 216, no. 1, pp. 293-300, 2011.

[29] G. Paxinos and C. Watson, The Rat Brain in Stereotaxic Coordinates, Academic Press, London, 2nd edition, 1986.

[30] A. L. Wang, Y. M. Liou, C. R. Pawlak, and Y. J. Ho, "Involvement of NMDA receptors in both MPTP-induced neuroinflammation and deficits in episodic-like memory in Wistar rats," Behavioural Brain Research, vol. 208, no. 1, pp. 38-46, 2010.
[31] H. N. Sy, S. L. Wu, W. F. Wang et al., "MPTP-induced dopaminergic degeneration and deficits in object recognition in rats are accompanied by neuroinflammation in the hippocampus," Pharmacology, Biochemistry, and Behavior, vol. 95, no. 2, pp. 158-165, 2010.

[32] M. H. Hsieh, S. C. Ho, K. Y. Yeh et al., "Blockade of metabotropic glutamate receptors inhibits cognition and neurodegeneration in an MPTP-induced Parkinson's disease rat model," Pharmacology, Biochemistry, and Behavior, vol. 102, no. 1, pp. 64-71, 2012.

[33] M. H. Hsieh, S. L. Gu, S. C. Ho et al., "Effects of MK-801 on recognition and neurodegeneration in an MPTP-induced Parkinson's rat model," Behavioural Brain Research, vol. 229, no. 1, pp. 41-47, 2012.

[34] M. A. Tikhonova, S. C. Ho, A. A. Akopyan et al., "Neuroprotective effects of ceftriaxone treatment on cognitive and neuronal deficits in a rat model of accelerated senescence," Behavioural Brain Research, vol. 330, pp. 8-16, 2017.

[35] L.-K. Song, K.-L. Ma, Y.-H. Yuan et al., "Targeted overexpression of $\alpha$-synuclein by rAAV2/1 vectors induces progressive nigrostriatal degeneration and increases vulnerability to MPTP in mouse," PLoS One, vol. 10, no. 6, article e0131281, 2015.

[36] S. K. Hota, K. Barhwal, K. Ray, S. B. Singh, and G. Ilavazhagan, "Ceftriaxone rescues hippocampal neurons from excitotoxicity and enhances memory retrieval in chronic hypobaric hypoxia," Neurobiology of Learning and Memory, vol. 89, no. 4, pp. 522-532, 2008.

[37] B. C. V. Campbell, Q.-X. Li, J. G. Culvenor et al., "Accumulation of insoluble $\alpha$-synuclein in dementia with Lewy bodies," Neurobiology of Disease, vol. 7, no. 3, pp. 192-200, 2000.

[38] W. J. Schulz-Schaeffer, "The synaptic pathology of alphasynuclein aggregation in dementia with Lewy bodies, Parkinson's disease and Parkinson's disease dementia," Acta Neuropathologica, vol. 120, no. 2, pp. 131-143, 2010.

[39] J. Burre, M. Sharma, and T. C. Sudhof, "Definition of a molecular pathway mediating $\alpha$-synuclein neurotoxicity," Journal of Neuroscience, vol. 35, no. 13, pp. 5221-5232, 2015.

[40] M. Ingelsson, "Alpha-synuclein oligomers-neurotoxic molecules in Parkinson's disease and other Lewy body disorders," Frontiers in Neuroscience, vol. 10, p. 408, 2016.

[41] M. Colom-Cadena, E. Gelpi, S. Charif et al., "Confluence of $\alpha$ synuclein, tau, and $\beta$-amyloid pathologies in dementia with Lewy bodies," Journal of Neuropathology \& Experimental Neurology, vol. 72, no. 12, pp. 1203-1212, 2013.

[42] E. Iseki, N. Takayama, W. Marui, K. Uéda, and K. Kosaka, "Relationship in the formation process between neurofibrillary tangles and Lewy bodies in the hippocampus of dementia with Lewy bodies brains," Journal of the Neurological Sciences, vol. 195, no. 1, pp. 85-91, 2002.

[43] Z. Esposito, L. Belli, S. Toniolo, G. Sancesario, C. Bianconi, and A. Martorana, "Amyloid $\beta$, glutamate, excitotoxicity in Alzheimer's disease: are we on the right track?," CNS Neuroscience of Therapeutics, vol. 19, no. 8, pp. 549-555, 2013.

[44] D. J. Selkoe, "Soluble oligomers of the amyloid beta-protein impair synaptic plasticity and behavior," Behavioural Brain Research, vol. 192, no. 1, pp. 106-113, 2008.

[45] G. Rammes, A. Hasenjäger, K. Sroka-Saidi, J. M. Deussing, and C. G. Parsons, "Therapeutic significance of NR2B-containing NMDA receptors and mGluR5 metabotropic glutamate receptors in mediating the synaptotoxic effects of $\beta$-amyloid 
oligomers on long-term potentiation (LTP) in murine hippocampal slices," Neuropharmacology, vol. 60, no. 6, pp. 982990, 2011.

[46] L. Texidó, M. Martín-Satué, E. Alberdi, C. Solsona, and C. Matute, "Amyloid $\beta$ peptide oligomers directly activate NMDA receptors," Cell Calcium, vol. 49, no. 3, pp. 184-190, 2011.

[47] W. Danysz and C. G. Parsons, “Alzheimer's disease, $\beta$-amyloid, glutamate, NMDA receptors and memantine - searching for the connections," British Journal of Pharmacology, vol. 167, no. 2, pp. 324-352, 2012.

[48] R. Gray, A. S. Rajan, K. A. Radcliffe, M. Yakehiro, and J. A. Dani, "Hippocampal synaptic transmission enhanced by low concentrations of nicotine," Nature, vol. 383, no. 6602, pp. 713-716, 1996.

[49] R. Lee Mosley, E. J. Benner, I. Kadiu et al., "Neuroinflammation, oxidative stress, and the pathogenesis of Parkinson's disease," Clinical Neuroscience Research, vol. 6, no. 5, pp. 261281, 2006.

[50] A. Plaitakis and P. Shashidharan, "Glutamate transport and metabolism in dopaminergic neurons of substantia nigra: implications for the pathogenesis of Parkinson's disease," Journal of Neurology, vol. 247, Supplement 2, pp. II25II35, 2000.

[51] P. Limousin, P. Krack, P. Pollak et al., "Electrical stimulation of the subthalamic nucleus in advanced Parkinson's disease," New England Journal of Medicine, vol. 339, no. 16, pp. 11051111, 1998.

[52] L. Capus, A. Melatini, M. Zorzon et al., "Chronic bilateral electrical stimulation of the subthalamic nucleus for the treatment of advanced Parkinson's disease," Neurological Sciences, vol. 22, no. 1, pp. 57-58, 2001.

[53] M. Luquin, L. Saldise, J. Guillen et al., "Does increased excitatory drive from the subthalamic nucleus contribute to dopaminergic neuronal death in Parkinson's disease?," Experimental Neurology, vol. 201, no. 2, pp. 407-415, 2006.

[54] H. Hall, S. Reyes, N. Landeck et al., "Hippocampal Lewy pathology and cholinergic dysfunction are associated with dementia in Parkinson's disease," Brain, vol. 137, no. 9, pp. 2493-2508, 2014.

[55] M. R. Hunsaker, J. A. Thorup, T. Welch, and R. P. Kesner, "The role of CA3 and CA1 in the acquisition of an objecttrace-place paired-associate task," Behavioral Neuroscience, vol. 120, no. 6, pp. 1252-6, 2006.

[56] H. A. Cameron and R. D. G. Mckay, "Adult neurogenesis produces a large pool of new granule cells in the dentate gyrus," The Journal of Comparative Neurology, vol. 435, no. 4, pp. 406-417, 2001.

[57] W. N. Zhang, H. H. J. Pothuizen, J. Feldon, and J. N. P. Rawlins, "Dissociation of function within the hippocampus: effects of dorsal, ventral and complete excitotoxic hippocampal lesions on spatial navigation," Neuroscience, vol. 127, no. 2, pp. 289-300, 2004.

[58] N. J. Broadbent, L. R. Squire, and R. E. Clark, "Spatial memory, recognition memory, and the hippocampus," Proceedings of the National Academy of Sciences of the United States of America, vol. 101, no. 40, pp. 14515-14520, 2004.

[59] I. G. McKeith, D. W. Dickson, J. Lowe et al., "Diagnosis and management of dementia with Lewy bodies: third report of the DLB consortium," Neurology, vol. 65, no. 12, pp. 18631872, 2005.
[60] D. H. Adamowicz, S. Roy, D. P. Salmon et al., "Hippocampal $\alpha$-synuclein in dementia with Lewy bodies contributes to memory impairment and is consistent with spread of pathology," The Journal of Neuroscience, vol. 37, no. 7, pp. 16751684, 2017.

[61] W. Yang and S. Yu, "Synucleinopathies: common features and hippocampal manifestations," Cellular and Molecular Life Sciences, vol. 74, no. 8, pp. 1485-1501, 2017.

[62] G. Battaglia, C. L. Busceti, G. Molinaro et al., "Endogenous activation of mGlu5 metabotropic glutamate receptors contributes to the development of nigro-striatal damage induced by 1-methyl-4-phenyl-1,2,3,6-tetrahydropyridine in mice," The Journal of Neuroscience, vol. 24, no. 4, pp. 828-835, 2004.

[63] L. Chen, J. Liu, U. Ali et al., "Blockade of mGluR5 reverses abnormal firing of subthalamic nucleus neurons in 6hydroxydopamine partially lesioned rats," The Chinese journal of physiology, vol. 54, no. 5, pp. 303-309, 2011.

[64] N. J. Maragakis, J. Dietrich, V. Wong et al., "Glutamate transporter expression and function in human glial progenitors," Glia, vol. 45, no. 2, pp. 133-143, 2004.

[65] A. Furuta, J. D. Rothstein, and L. J. Martin, “Glutamate transporter protein subtypes are expressed differentially during rat CNS development," The Journal of Neuroscience, vol. 17, no. 21, pp. 8363-8375, 1997.

[66] O. Bar-Peled, H. Ben-Hur, A. Biegon et al., "Distribution of glutamate transporter subtypes during human brain development," Journal of Neurochemistry, vol. 69, no. 6, pp. 25712580, 1997.

[67] K. Kim, S. G. Lee, T. P. Kegelman et al., "Role of excitatory amino acid transporter-2 (EAAT2) and glutamate in neurodegeneration: opportunities for developing novel therapeutics," Journal of Cellular Physiology, vol. 226, no. 10, pp. 24842493, 2011.

[68] J. D. Rothstein, M. Dykes-Hoberg, C. A. Pardo et al., "Knockout of glutamate transporters reveals a major role for astroglial transport in excitotoxicity and clearance of glutamate," Neuron, vol. 16, no. 3, pp. 675-686, 1996.

[69] Y. Kanai and M. A. Hediger, "The glutamate and neutral amino acid transporter family: physiological and pharmacological implications," European Journal of Pharmacology, vol. 479, no. 1-3, pp. 237-247, 2003.

[70] S. S. Huang, J. He, D. M. Zhao, X. Y. Xu, H. P. Tan, and H. Li, "Effects of mutant huntingtin on mGluR5-mediated dual signaling pathways: implications for therapeutic interventions," Cellular and Molecular Neurobiology, vol. 30, no. 7, pp. 1107-1115, 2010.

[71] D. Neef and A. D. Walling, "Dementia with Lewy bodies: an emerging disease," American Family Physician, vol. 73, no. 7, pp. 1223-1229, 2006.

[72] S.-T. Tsai, W.-Y. Chuang, C.-C. Kuo et al., "Dorsolateral subthalamic neuronal activity enhanced by median nerve stimulation characterizes Parkinson's disease during deep brain stimulation with general anesthesia," Journal of Neurosurgery, vol. 123, no. 6 , pp. 1394-1400, 2015. 


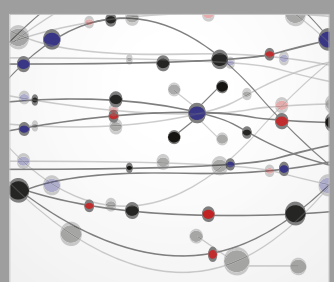

The Scientific World Journal
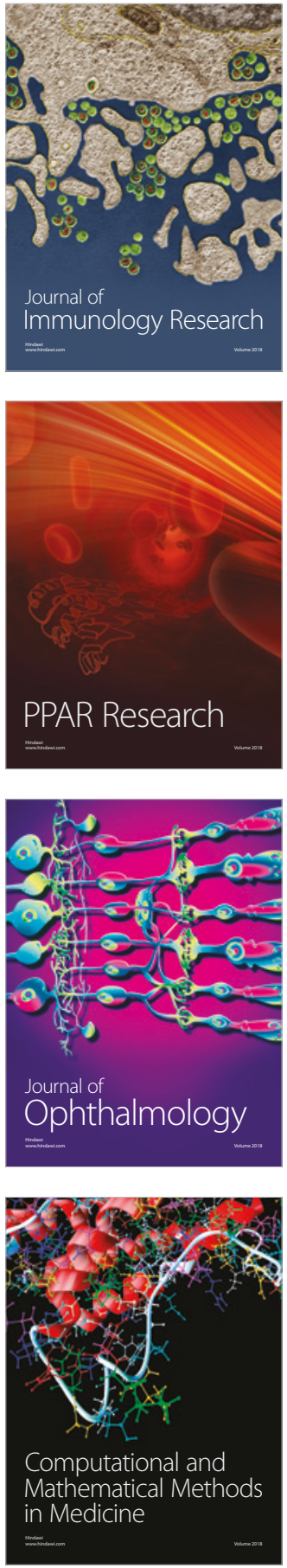

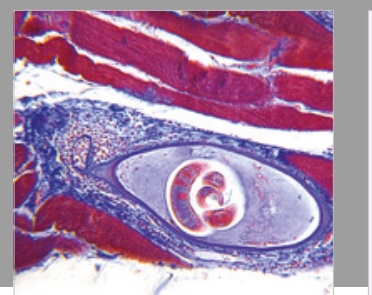

Gastroenterology Research and Practice

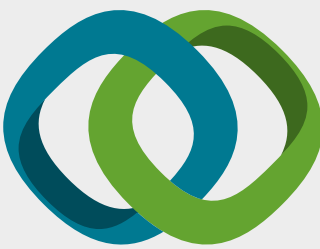

\section{Hindawi}

Submit your manuscripts at

www.hindawi.com
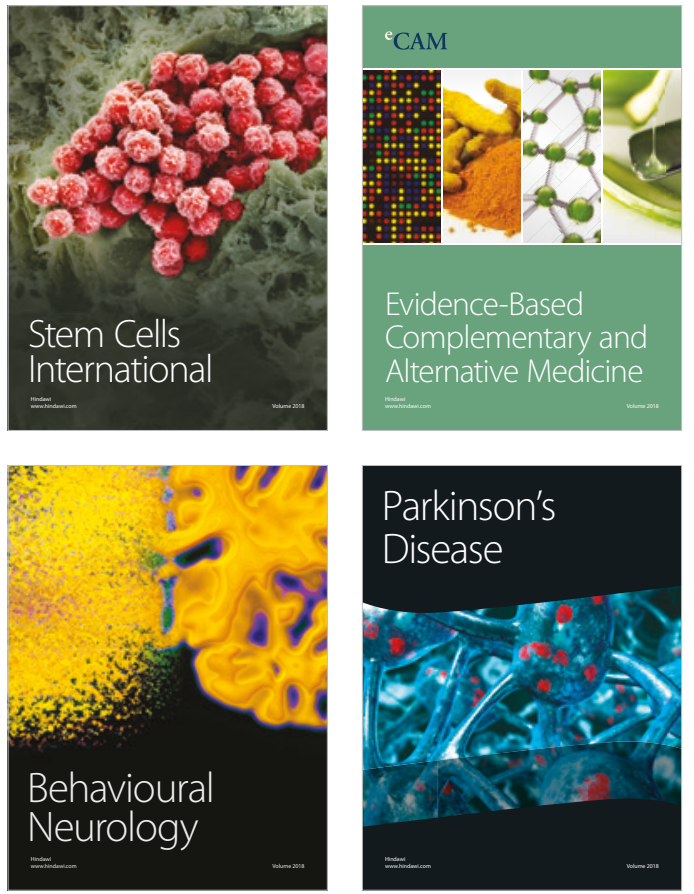

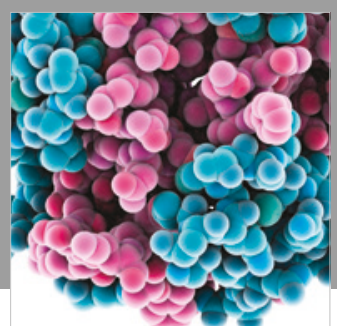

ournal of

Diabetes Research

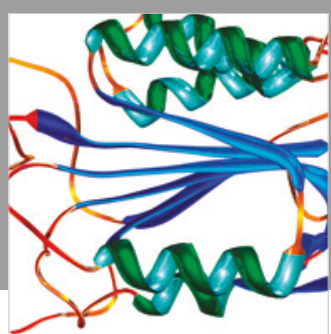

Disease Markers
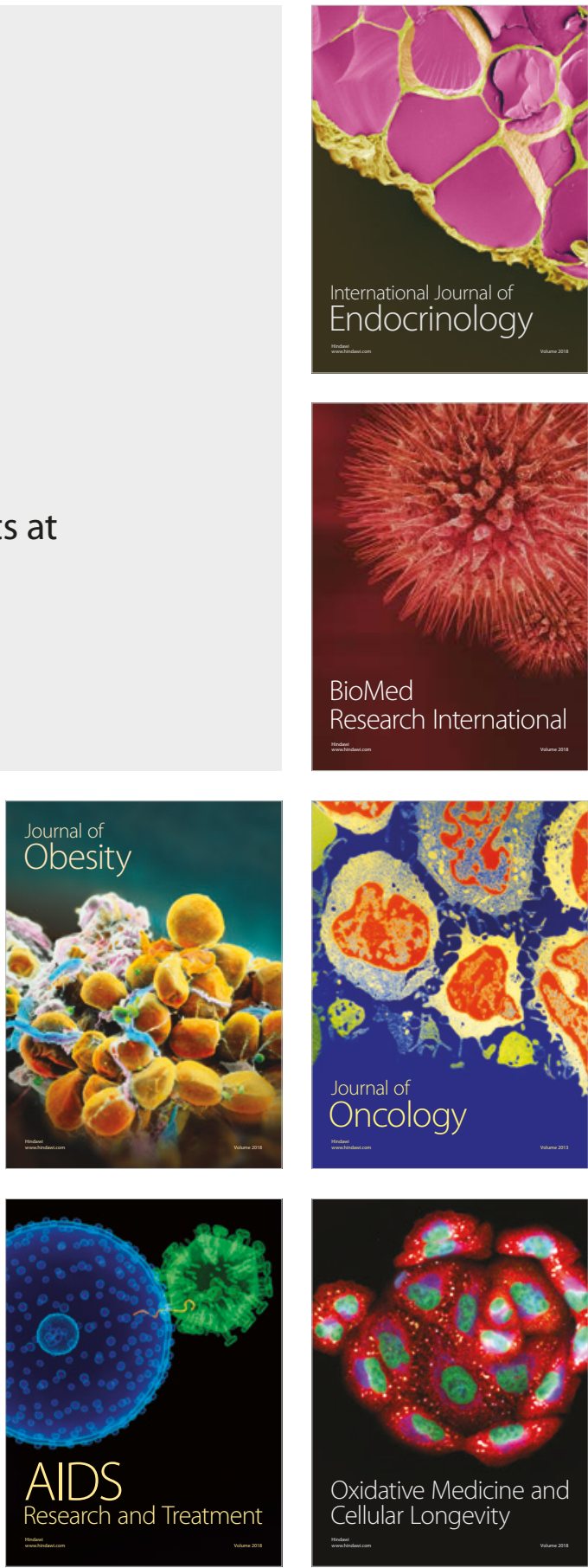